Pontifícia Universidade Católica

DO RIO DE JANEIRO

Sueli Tiomno Tolmasquim

\title{
PROJETO E CONTROLE DA OPERAÇÃO DE PASSAGEM DE PIGS EM DUTOS
}

Dissertação apresentada ao Programa de Pós-graduação em Engenharia Mecânica da PUC-Rio como requisito parcial para obtenção do título de Mestre em Ciências de Engenharia Mecânica.

Orientadora: Angela Ourivio Nieckele 


\title{
Sueli Tiomno Tolmasquim
}

\section{Projeto e Controle da Operação de Passagem de Pigs em Dutos}

\begin{abstract}
Dissertação apresentada como requisito parcial para obtenção do grau de Mestre pelo Programa de Pósgraduação em Engenharia Mecânica da PUC-Rio. Aprovada pela Comissão Examinadora abaixo assinada.
\end{abstract}

\author{
Prof ${ }^{\mathrm{a}}$. Angela Ourivio Nieckele \\ Orientadora \\ Departamento de Engenharia Mecânica - PUC-Rio \\ Prof. Luis Fernando Alzuguir Azevedo \\ Departamento de Engenharia Mecânica - PUC-Rio \\ Prof. Sidney Stuckenbruck \\ Departamento de Engenharia Mecânica - PUC-Rio \\ Prof. José Eugênio Leal \\ Coordenador Setorial do Centro \\ Técnico Científico - PUC-Rio
}


Todos os direitos reservados. É proibida a reprodução total ou parcial do trabalho sem autorização da universidade, do autor e do orientador.

\section{Sueli Tiomno Tolmasquim}

Graduou-se em Engenharia Mecânica pela UFRJ (Universidade Federal do Rio de Janeiro) em 1986. Ingressou na Petrobras em 1989, e desde então tem atuado na área de transporte dutoviário, com ênfase em estudos hidráulicos. Possui trabalhos apresentados em congressos no Brasil e no exterior, em tecnologia de dutos. Atualmente, é responsável pela Coordenadoria de Dutos da Gerência de Tecnologia da PETROBRAS Transportes - Transpetro.

Ficha Catalográfica

Tolmasquim, Sueli Tiomno

Projeto e controle da operação de passagem de pigs em dutos / Sueli Tiomno Tolmasquim ; orientadora: Angela Ourivio Nieckele. - Rio de Janeiro : PUC, Departamento de Engenharia Mecânica, 2004.

109 f. : il. ; $30 \mathrm{~cm}$

Dissertação (mestrado) - Pontifícia Universidade Católica do Rio de Janeiro, Departamento de Engenharia Mecânica.

Inclui referências bibliográficas.

1. Engenharia mecânica - Teses. 2. Simulação transiente. 3. Pig. 4. Deslocamento de óleo. 5. Gás-líquido. 6. Controle de Processo I. Nieckele, Angela Ourivio. II. Pontifícia Universidade Católica do Rio de Janeiro. Departamento de Engenharia Mecânica. III. Título. 
Aos meus pais Silvia e Ernesto que sempre foram uma referência na minha formação profissional. 


\section{Agradecimentos}

À minha orientadora, Professora Angela Ourivio Nieckele, pela orientação, aprendizado, atenção e parceria, durante a realização das cadeiras de mestrado e na elaboração da dissertação.

À PETROBRAS Transportes S.A. - TRANSPETRO - por ter aprovado e apoiado a realização deste mestrado.

Ao meu namorado Leo Melo por todo o carinho, paciência e incentivo.

Aos meus familiares e amigos pela compreensão durante este período e à minha mãe Silvia pela educação, carinho e dedicação em todas as horas.

Aos meus colegas de trabalho Richard Ward, Marcelino Gomes e Lino Moreira, que como gerentes da Transpetro, me incentivaram e deram o suporte necessário.

Aos colegas de trabalho Luciano Macedo Josino da Costa, Antônio Geraldo de Souza, Ricardo Dias e Helmut Kossatz pela troca de idéias durante o desenvolvimento da dissertação e revisão do texto.

Ao Prof. Luis Fernando A. Azevedo pela orientação na escolha do tema da dissertação e ao longo do curso de mestrado.

Aos colegas Cláudio Veloso Barreto pela ajuda na interface gráfica e Luiz Fernando Pires no módulo interativo.

Ao Departamento de Engenharia Mecânica da PUC-Rio.

Aos meus amigos Marcos Jangelavicin, Henriete Seixas, Luiz Antônio, Marcius Ferrari e Ricardo Dias que compartilharam comigo esta jornada.

Aos professores que participaram da Comissão examinadora.

À todos que não foram citados acima, porém que tiveram participação na elaboração da minha dissertação. 


\section{Resumo}

Tolmasquim, Sueli Tiomno. Projeto e Controle da Operação de Passagem de Pigs em Dutos. Rio de janeiro, 2004, 109 p. Dissertação de Mestrado Departamento de Engenharia Mecânica, Pontifícia Universidade Católica do Rio de Janeiro.

$\mathrm{Na}$ indústria do petróleo, a passagem de pigs em dutos tem sido largamente aplicada com diferentes propósitos: limpeza do tubo, inspeção, remoção de líquido e separação de produtos, entre outros. A eficiência e segurança de uma operação com pig demandam que diversos parâmetros operacionais, tais como pressões máximas e mínimas no duto e velocidade de movimentação do pig, sejam bem avaliados durante a etapa de planejamento e mantidos dentro de determinados limites durante o acompanhamento da operação. Tendo como objetivo a obtenção de uma ferramenta eficiente para ajudar no controle e projeto das operações de passagem de pigs, desenvolveu-se um código numérico baseado no método de diferenças finitas para a simulação de escoamentos transientes de dois fluidos, podendo estes ser líquido-líquido, gás-gás ou líquido-gás. Módulos para controle automático das variáveis do processo foram incluídos, visando à previsão do escoamento mediante diferentes estratégias para alcançar uma operação eficiente. Problemas teste foram realizados, validando a metodologia. Por fim, os resultados obtidos com o simulador são comparados com um caso real de esvaziamento de um trecho do oleoduto OSPAR, pertencente à Petrobras, com 30" de diâmetro e extensão de 60 km.

\section{Palavras-chave}

simulação transiente; pig; deslocamento de óleo; gás-líquido; controle de processo. 


\section{Abstract}

Tolmasquim, Sueli Tiomno. Design and Control of Pig Operations Through Pipelines. Rio de janeiro, 2004, 109 p. MSc. Dissertation Departamento de Engenharia Mecânica, Pontifícia Universidade Católica do Rio de Janeiro.

In the oil industry, pigging operations in pipelines have been largely applied for different purposes: pipe cleaning, inspection, liquid removal and product separation, among others. Pigging operations to be efficient and safe require a number of operational parameters, such as maximum and minimum pressures in the pipeline and pig velocity, to be well evaluated during planning stage and maintained within stipulated limits while the operation accomplishment. With the objective of providing an efficient tool to assist in the controlling and designing of pig operations through pipelines, a numerical code based on a finite difference scheme for a two-fluid transient flow simulation was developed. The model accounts for liquid-liquid, gas-gas and liquid-gas products in the pipeline. Automatic control modules for some process variables were included to permit the flow prediction by means of different strategies to reach an efficient operation. Test problems were performed to validate the methodology. At last, simulation results were compared with an actual liquid displacement operation at a $60 \mathrm{~km}$ segment of the 30" diameter OSPAR pipeline, owned by Petrobras.

\section{Keywords:}

transient simulation; pig; oil displacement; gas-liquid; process control. 


\section{Sumário}

1. Introdução 18

1.1. Motivação 23

1.2. Revisão bibliográfica $\quad 24$

1.2.1. Softwares Comerciais 24

1.2.2. Movimentação de Pig e Modelos Computacionais 26

1.2.3. Simplificação da Análise Hidráulica 28

1.3. Objetivo 28

1.4. Organização do Trabalho 29

2. Modelamento Matemático 31

2.1. Conservação de Massa 32

2.1.1. Variações da Área da Tubulação 32

2.1.2. Compressibilidade do Fluido 33

2.1.3. Equação da Pressão 34

2.2. Conservação da Quantidade de Movimento Linear 34

2.2.1. Fator de Atrito 35

2.3. Propriedades do Fluido 36

2.3.1. Massa Específica 36

2.3.2. Viscosidade Absoluta 37

2.4. Condições de Contorno 38

2.5. Inicialização 39

2.5.1. Fluido em Repouso 39

2.5.2. Inicialização em Regime Permanente 40

2.6. Modelagem da Dinâmica do Pig 40

2.7. Pressão Máxima de Operação Admissível (PMOA) 41

2.8. Controle de Processos 44

2.8.1. Controlador PID 46

2.8.2. Ação do Controlador 47

2.8.3. Controle de Duas Variáveis do Processo 47 
3. Método Numérico 49

3.1. Sistema de Coordenadas Móvel 49

3.2. Discretização das Equações 51

3.2.1. Equação da Continuidade 52

3.2.2. Equação da Quantidade do Movimento Linear $\quad 53$

3.3. Discretização da Equação Diferencial de um Controlador PID 55

3.4. Solução do Sistema Algébrico 56

3.5. Critério de Convergência 58

3.5.1. Critérios Avaliados 59

3.6. Controle da Operação 60

3.6.1. Malha Aberta 60

3.6.2. Controle Automático 60

3.6.3. Simulação Interativa 61

3.7. Fluxograma do Modelo 61

4. Estudo de Casos 65

4.1. Exemplo 1 - Controle da Velocidade do Pig e Pressão Mínima 65

4.1.1. Situação sem Controle Automático da Válvula na Saída 66

4.1.2. Situação com Controle Automático da Válvula na Saída $\quad 70$

4.2. Exemplo - Controle da Velocidade do Pig e Pressão Mínima

4.2.1. Situação sem Controle Automático da Válvula na Saída $\quad 76$

4.2.2. Situação com Controle Automático da Válvula na Saída 79

4.2.3. Situação com Controle Automático da Válvula na Entrada

4.3. Aplicação do Modelo Numérico a um Caso Real de Esvaziamento de Oleoduto 
4.3.1. Descrição da Operação

4.3.2. Validação do Modelo Numérico 90

4.3.3. Otimização da Operação de Esvaziamento do Óleo 96

5. Comentários Finais 103

5.1. Recomendações para Futuros Estudos 104

6. Referências Bibliográficas 105 


\section{Lista de Figuras}

Figura 1.1 - Perfil típico de um trecho de oleoduto 19

Figura 1.2 - Vista lateral de um flexipig de 30" 21

Figura 1.3 - Vista posterior de um flexipig de 30" 21

Figura 1.4 - Escoamento em um ponto alto de um duto com coluna abertura 22

Figura 1.5 - Gradiente hidráulico com e sem abertura de coluna 22

Figura 2.1 - Volume de controle para análise de escoamento unidimensional transiente 31

Figura 2.2 - Representação esquemática das pressões em oleodutos, ou trecho de oleoduto, de mesma espessura e material 43

Figura 2.3 - Sistema de controle em malha aberta 45

Figura 2.4 - Sistema de controle em malha fechada 45

Figura 2.5 - Controle de duas variáveis do processo por "passa baixo" 48

Figura 3.1 - Distribuição deslocada da malha 51

Figura 3.2 - Sistema algébrico pentadiagonal 57

Figura 3.3 - Fluxograma do programa 63

Figura 3.4 - Fluxograma da subrotina MARCHA 64

Figura 4.1 - Variação da pressão com o tempo - sem controle. Exemplo 1

Figura 4.2 - Variação da velocidade do pig em função da sua posição - sem controle. Exemplo 1

Figura 4.3 - Posição do pig com o tempo - sem controle. Exemplo 1

Figura 4.4 - Percentual de abertura da válvula de controle com o tempo. Exemplo 1. 
Figura 4.5 - Variação da pressão com o tempo - com controle automático. Exemplo 1.

Figura 4.6- Variação da velocidade do pig com o tempo - com controle automático. Exemplo 1.

Figura 4.7 - Posição do pig com o tempo - com controle automático. Exemplo 1.

Figura 4.8 - Perfil do duto. Exemplo 2

Figura 4.9 - Variação da pressão com o tempo - sem controle. Exemplo 2.

Figura 4.10 - Variação da velocidade do pig com o tempo - sem controle. Exemplo 2.

Figura 4.11 - Posição do pig com o tempo - sem controle. Exemplo 2

Figura 4.12 - Percentual de abertura da válvula de controle de saída com o tempo. Exemplo 2.

Figura 4.13 - Variação da velocidade do pig com o tempo - com controle automático da válvula de saída. Exemplo 2.

Figura 4.14 - Variação da pressão com o tempo - com controle automático da válvula de saída. Exemplo 2.

Figura 4.15 - Variação da pressão com o tempo - com controle automático da válvula de entrada. Exemplo 2.

Figura 4.16 - Variação da velocidade do pig com o tempo - com controle automático da válvula de entrada. Exemplo 2

Figura 4.17 - Percentual de abertura da válvula de controle da entrada com o tempo. Exemplo 2.

Figura 4.18 - Perfil Planialtimétrico do OSPAR.

Figura 4.19 - Lançador de pig do OSPAR na REPAR 88

Figura 4.20 - Armazenamento e sistema de aquecimento de $\mathrm{N}_{2}$.

Figura 4.21 - Módulo emissor de sinal para localização do pig.

Figura 4.22 - Módulo receptor de sinal para localização do pig.

Figura 4.23 - Perfil e PMOA do trecho REPAR-Itararé

Figura 4.24 - Variação da pressão medida na REPAR com o tempo 
Figura 4.25 - Variação do nível do tanque de recebimento de petróleo em SFS com o tempo

Figura 4.26 - Variação da vazão mássica do tanque de recebimento de petróleo em SFS com o tempo

Figura 4.27 - Comparação entre pressões medidas e simuladas em Itararé

Figura 4.28 - Comparação entre vazão mássica medida e simulada na REPAR

Figura 4.29 - Comparação entre a posição do pig medida e simulada

Figura 4.30 - Variação da pressão na entrada com o tempo - com válvula de controle. OSPAR.

Figura 4.31 - Variação da pressão na saída com o tempo - com válvula de controle - OSPAR

Figura 4.32 - Variação da pressão na entrada, saída e ponto de cota máxima com o tempo - com válvula de controle OSPAR

Figura 4.33 - Variação da pressão ao longo da tubulação em $\mathrm{t}=390 \mathrm{~s}$, quando a pressão mínima foi atingida. OSPAR

Figura 4.34 - Variação da velocidade do pig em função da sua posição - com válvula de controle. OSPAR.

Figura 4.35 - Velocidade do pig com o tempo. - com válvula de controle. OSPAR.

Figura 4.36 - Posição do pig com o tempo. - com válvula de controle. OSPAR.

Figura 4.37 - Variação da vazão na saída com o tempo.- com válvula de controle OSPAR.

Figura 4.38 - Variação do percentual de abertura da válvula de controle na saída com o tempo. OSPAR. 


\section{Lista de tabelas}

Tabela 2.1 - Aplicações típicas dos algoritmos PID na indústria 47

Tabela 4.1 - Parâmetros dos controladores. Exemplo 1

Tabela 4.2 - Coordenadas do duto. Exemplo 2

Tabela 4.3 - Parâmetros dos Controladores da Velocidade do Pig. Exemplo $2 \quad 79$

Tabela 4.4 - Parâmetros dos Controladores da Pressão Máxima. Exemplo 2

Tabela 4.5 - Força de contato entre o pig e a parede do tubo 91

Tabela 4.6 - Parâmetros dos Controladores. OSPAR. 97 


\section{Nomenclatura}

$a \quad$ Velocidade do som em um meio infinito isotérmico $(\mathrm{m} / \mathrm{s})$

$A \quad$ Área da seção transversal

AC Ação do controlador

c Velocidade de propagação da onda acústica na tubulação $(\mathrm{m} / \mathrm{s})$

$c_{\mu, p} \quad$ Coeficiente de pressão da viscosidade

D Diâmetro do tubo (m)

$D_{e} \quad$ Diâmetro externo do tubo (m)

$e \quad$ Espessura da parede do tudo (m)

e Erro do controlador

E Módulo de Young

E Fator devido ao tipo de solda longitudinal

$f \quad$ Coeficiente de atrito

F $\quad$ Força

$F \quad$ Fator de projeto

$F_{\text {th }} \quad$ Fator de teste de pressão

$F_{a t}\left(V_{p}\right)$ Força de contato entre o pig e a parede do tubo $\left(\mathrm{Kg} \cdot \mathrm{m} / \mathrm{s}^{2}\right)$

$g \quad$ Aceleração da gravidade $\left(\mathrm{m} / \mathrm{s}^{2}\right)$

$h \quad$ Fator de escala associado à coordenada móvel

$h u \quad$ Fator de escala associado ao volume deslocado

K Ganho do controlador

$m \quad$ Massa do pig $(\mathrm{kg})$

$\dot{m} \quad$ Vazão mássica $(\mathrm{kg} / \mathrm{s})$

$\bar{M} \quad$ Massa molecular da mistura gasosa

$n \quad$ Tempo discreto

$P \quad$ Pressão

$P_{\text {mopth }} \quad$ Pressão máxima de operação devido ao teste de hidrostático

$P_{\text {proj }} \quad$ Pressão de projeto 


\begin{tabular}{|c|c|}
\hline$P_{t h}$ & Pressão exercida no teste hidrostático \\
\hline$P_{1}$ & Pressão na face à montante do pig $(\mathrm{Pa})$ \\
\hline$P_{2}$ & Pressão na face à jusante do pig $(\mathrm{Pa})$ \\
\hline$R$ & Constante do gás $(\mathrm{N} \cdot \mathrm{m} / \mathrm{Kg} \cdot \mathrm{K})$ \\
\hline$\Re$ & Constante universal dos gases $(\mathrm{J} / \mathrm{kgmol} \cdot \mathrm{K})$ \\
\hline$s$ & Coordenada axial \\
\hline$S$ & Tensão mínima de escoamento do material $(\mathrm{Pa})$ \\
\hline$S P$ & Set point do controle da variável de processo \\
\hline$t$ & Tempo (s) \\
\hline$T$ & Fator devido à temperatura \\
\hline$T_{D}$ & Tempo derivativo (s) \\
\hline$T_{I}$ & Tempo integral (s) \\
\hline$T_{o}$ & Tempo de amostragem (s) \\
\hline$u$ & Saída do controlador \\
\hline$V$ & Velocidade $(\mathrm{m} / \mathrm{s})$ \\
\hline$\tilde{V}$ & Velocidade relativa $(\mathrm{m} / \mathrm{s})$ \\
\hline$V P$ & Variável de processo \\
\hline$z$ & Elevação (m) \\
\hline$z$ & Fator de compressibilidade \\
\hline \multicolumn{2}{|c|}{ Letras Gregas: } \\
\hline$\chi$ & Percentagem de abertura da válvula \\
\hline$\varepsilon$ & Rugosidade do tubo (m) \\
\hline$\eta$ & Coordenada computacional axial \\
\hline$\mu$ & Coeficiente de Poisson \\
\hline$\mu_{f}$ & Viscosidade absoluta $(\mathrm{kg} / \mathrm{m} \cdot \mathrm{s})$ \\
\hline$\theta$ & Ângulo do eixo do tubo com a horizontal \\
\hline$\rho$ & Massa específica $\left(\mathrm{kg} / \mathrm{m}^{3}\right)$ \\
\hline$\tau_{0}$ & Tensão viscosa \\
\hline
\end{tabular}




\section{Subscritos:}

$\begin{array}{ll}\text { atm } & \text { Atmosférica } \\ \text { din } & \text { Dinâmica } \\ i & \text { Ponto nodal } \\ \text { in } & \text { Entrada do duto } \\ g & \text { Malha } \\ m & \text { Média } \\ \text { out } & \text { Saída do duto } \\ p & \text { Pig } \\ r e f & \text { Variáveis de referência } \\ \text { stat } & \text { Estática }\end{array}$

\section{Sobrescritos:}

neg Sentido negativo

pos Sentido positivo 